\title{
Nonexistence of Large Nuclei in the Liquid Drop Model
}

\author{
RUPERT L. FRANK ${ }^{1}$, ROWAN KILLIP ${ }^{2}$ and PHAN THÀNH NAM ${ }^{3}$ \\ ${ }^{1}$ Mathematics 253-37, Caltech, Pasadena, CA 91125, USA. e-mail: rlfrank@caltech.edu \\ ${ }^{2}$ UCLA Mathematics Department, Box 951555, Los Angeles, CA 90095-1555, USA. \\ e-mail:killip@math.ucla.edu \\ ${ }^{3}$ Institute of Science and Technology Austria, Am Campus 1, 3400 Klosterneuburg, Austria. \\ e-mail: pnam@ist.ac.at
}

Received: 18 April 2016 / Revised: 7 May 2016 / Accepted: 9 May 2016

Published online: 14 June 2016 - (C) The Author(s) 2016. This article is published with open access at Springerlink.com

\begin{abstract}
We give a simplified proof of the nonexistence of large nuclei in the liquid drop model and provide an explicit bound. Our bound is within a factor of 2.3 of the conjectured value and seems to be the first quantitative result.
\end{abstract}

Mathematics Subject Classification. 49Q10, 49Q20, 81V35.

Keywords. liquid drop model, minimization problem, nonexistence.

We consider the minimization problem

$$
E(A)=\inf \{\mathcal{E}(\Omega):|\Omega|=A\}
$$

over all measurable set $\Omega \subset \mathbb{R}^{3}$ with the energy functional

$$
\mathcal{E}[\Omega]=\operatorname{Per} \Omega+\frac{1}{2} \iint_{\Omega \times \Omega} \frac{\mathrm{d} x \mathrm{~d} y}{|x-y|} .
$$

Here $\Omega$ can be interpreted as a nucleus in the liquid drop model with density 1 , and hence the volume $|\Omega|=A$ is the number of nucleons (protons and neutrons) in the nucleus. Mathematically, $A$ is not necessarily an integer. The perimeter Per $\Omega$ is taken in the sense of De Giorgi, namely

$$
\operatorname{Per} \Omega=\sup \left\{\int_{\Omega} \operatorname{div} F(x) \mathrm{d} x\left|F \in C_{0}^{1}\left(\mathbb{R}^{3}, \mathbb{R}^{3}\right),\right| F \mid \leq 1\right\},
$$

which boils down to the surface area of $\Omega$ when the boundary is smooth. The Coulomb term describes the proton repulsion in the nucleus, where the proton charge has been normalized appropriately. The liquid drop model goes back to the pioneering works of Gamow [9], von Weizsäcker [16] and Bohr [2] in 1930s, and 
recently it has gained renewed interest from many authors, see for instance [1,3$6,8,10-12]$.

It is well known that among all measurable sets of a given volume, balls minimize the perimeter (by the isoperimetric inequality [7]) and maximize the Coulomb self-interaction energy (by the Riesz rearrangement inequality [15]). This energy competition makes the liquid drop model highly nontrivial. It is generally assumed in the physics literature and conjectured in the mathematics literature [6] that $E(A)$ is minimized by a ball up to

$$
A_{c}=\frac{2-2^{2 / 3}}{2^{2 / 3}-1} \cdot \frac{|B| \operatorname{Per} B}{\frac{1}{2} \iint_{B \times B}|x-y|^{-1} \mathrm{~d} x \mathrm{~d} y}=5 \cdot \frac{2-2^{2 / 3}}{2^{2 / 3}-1} \approx 3.518,
$$

(see also [8]) and that for $A>A_{c}$ there is no minimizer.

The fact that there is no minimizer for large $A$ has been shown only recently in remarkable works of Knüpfer-Muratov [10] and Lu-Otto [12]. Their methods are inspired by techniques from geometric measure theory and seem to lead to rather large constants. In the present paper, we will provide a direct and simple proof of the nonexistence and give an explicit bound on the maximal size of a nucleus. Our main result is

THEOREM. If $A>8$, then $E(A)$ has no minimizer.

This is within a factor of 2.3 of the conjectured value and seems to be the first quantitative result. We also emphasize that balls are locally stable up to $A=10$ [3]. Our proof builds on ideas in [12,14], which were originally developed to deal with the nonexistence in the Thomas-Fermi-Dirac-von Weisäcker theory.

For every $\nu \in \mathbb{S}^{2}$ and $\ell \in \mathbb{R}$ we consider the plane

$$
H_{v, \ell}:=\left\{x \in \mathbb{R}^{3} \mid v \cdot x=\ell\right\}
$$

as well as the half-spaces

$$
H_{v, \ell}^{+}:=\left\{x \in \mathbb{R}^{3} \mid v \cdot x>\ell\right\}, \quad H_{v, \ell}^{-}:=\left\{x \in \mathbb{R}^{3} \mid v \cdot x<\ell\right\} .
$$

For a set $\Omega \subset \mathbb{R}^{3}$ we denote

$$
\Omega_{v, \ell}^{ \pm}=\Omega \cap H_{v, \ell}^{ \pm} .
$$

We use the following simple result from the theory of sets of finite perimeter.

LEMMA. Let $\Omega \subset \mathbb{R}^{3}$ have finite perimeter and $v \in \mathbb{S}^{2}$. Then for almost every $\ell \in \mathbb{R}$,

$$
\operatorname{Per} \Omega_{v, \ell}^{+}+\operatorname{Per} \Omega_{v, \ell}^{-}=\operatorname{Per} \Omega+2 \mathcal{H}^{2}\left(\Omega \cap H_{v, \ell}\right) .
$$

Here $\mathcal{H}^{2}$ denotes the two-dimensional Hausdorff measure.

Proof of Lemma. For a set $E \subset \mathbb{R}^{3}$ of finite perimeter and its characteristic function $\chi_{E}$ we consider the measure $\mu_{E}=-\nabla \chi_{E}$ and note that $\operatorname{Per} E=\left|\mu_{E}\right|\left(\mathbb{R}^{3}\right)$. 
According to [13, Ex. 15.13], for almost every $\ell \in \mathbb{R}$,

$$
\mu_{\Omega_{v, \ell}^{-}}=\left.\mu_{\Omega}\right|_{H_{v, \ell}^{-}}+\left.v \mathcal{H}^{2}\right|_{\Omega \cap H_{v, \ell}} .
$$

As in the proof of [13, Lem. 15.12], the measures on the right side are mutually singular and therefore

$$
\left|\mu_{\Omega_{v, \ell}^{-}}\right|=\left.\left|\mu_{\Omega}\right|\right|_{H_{v, \ell}^{-}}+\left.\mathcal{H}^{2}\right|_{\Omega \cap H_{v, \ell}} .
$$

Thus,

$$
\operatorname{Per} \Omega_{v, \ell}^{-}=\left|\mu_{\Omega}\right|\left(H_{v, \ell}^{-}\right)+\mathcal{H}^{2}\left(\Omega \cap H_{v, \ell}\right) .
$$

Adding this and the corresponding equality for $-v$ and $-\ell$ we obtain the lemma.

Proof of Theorem. Let $\Omega$ be a minimizer for $E(A)$ for some $A>0$. By minimality of $\Omega$ and subadditivity of $E$, we have for every $v \in \mathbb{S}^{2}$ and $\ell \in \mathbb{R}$,

$$
\mathcal{E}\left(\Omega_{v, \ell}^{+}\right)+\mathcal{E}\left(\Omega_{v, \ell}^{-}\right) \geq E\left(\left|\Omega_{v, \ell}^{+}\right|\right)+E\left(\left|\Omega_{v, \ell}^{-}\right|\right) \geq E(A)=\mathcal{E}(\Omega) .
$$

By the lemma, for almost every $\ell \in \mathbb{R}$ this is the same as

$$
2 \mathcal{H}^{2}\left(\Omega \cap H_{v, \ell}\right) \geq \iint_{H_{v, \ell}^{+} \times H_{v, \ell}^{-}} \frac{\chi_{\Omega}(x) \chi_{\Omega}(y)}{|x-y|} \mathrm{d} x \mathrm{~d} y .
$$

We integrate this inequality over $\ell \in \mathbb{R}$ and use the fact that

$$
\int_{\mathbb{R}} \mathcal{H}^{2}\left(\Omega \cap H_{\nu, \ell}\right) \mathrm{d} \ell=|\Omega|=A
$$

(by Fubini's theorem) and

$$
\int_{\mathbb{R}} \chi_{\{v \cdot x>\ell>v \cdot y\}} \mathrm{d} \ell=(v \cdot(x-y))_{+},
$$

to get

$$
2 A \geq \iint_{\mathbb{R}^{3} \times \mathbb{R}^{3}} \chi_{\Omega}(x) \frac{(\nu \cdot(x-y))_{+}}{|x-y|} \chi_{\Omega}(y) \mathrm{d} x \mathrm{~d} y .
$$

Finally, we average the bound with respect to $v \in \mathbb{S}^{2}$ and use the fact that, for any $a \in \mathbb{R}^{3}$,

$$
(4 \pi)^{-1} \int_{\mathbb{S}^{2}}(\nu \cdot a)_{+} \mathrm{d} \nu=\frac{|a|}{2} \int_{0}^{\pi / 2} \cos \theta \sin \theta \mathrm{d} \theta=\frac{|a|}{4}
$$

to conclude that

$$
2 A \geq \frac{1}{4} \iint_{\mathbb{R}^{3} \times \mathbb{R}^{3}} \chi_{\Omega}(x) \chi_{\Omega}(y) \mathrm{d} x \mathrm{~d} y=\frac{A^{2}}{4} .
$$

Thus, $A \leq 8$, which proves the theorem. 


\section{Acknowledgements}

Open access funding provided by Institute of Science and Technology Austria. Phan Thành Nam would like to thank $\mathrm{H}$. Van Den Bosch for helpful discussions. Partial support by US National Science Foundation DMS-1363432 (R.L.F.), DMS-1265868 (R.K.) and Austrian Science Fund (FWF) Project Nr. P 27533-N27 (P.T.N.) are acknowledged.

Open Access This article is distributed under the terms of the Creative Commons Attribution 4.0 International License (http://creativecommons.org/licenses/by/4.0/), which permits unrestricted use, distribution, and reproduction in any medium, provided you give appropriate credit to the original author(s) and the source, provide a link to the Creative Commons license, and indicate if changes were made.

\section{References}

1. Alberti, G., Choksi, R., Otto, F.: Uniform energy distribution for an isoperimetric problem with long-range interactions. J. Am. Math. Soc. 22, 596-605 (2009)

2. Bohr, N.: Neutron capture and nuclear constitution. Nature 137, 344-348 (1936)

3. Bonacini, M., Cristoferi, R.: Local and global minimality results for a nonlocal isoperimetric problem on $\mathbb{R}^{N}$. SIAM J. Math. Anal. 46(4), 2310-2349 (2014)

4. Cicalese, M., Spadaro, E.: Droplet minimizers of an isoperimetric problem with longrange interactions. Commun. Pure Appl. Math. 66, 1298-1333 (2013)

5. Choksi, R., Peletier, M.A.: Small volume fraction limit of the diblock copolymer problem: I. Sharp-interface functional. SIAM J. Math. Anal. 42, 1334-1370 (2010)

6. Choksi, R., Peletier, M.A.: Small volume-fraction limit of the diblock copolymer problem: II. Diffuse-interface functional. SIAM J. Math. Anal. 43(2), 739-763 (2011)

7. De Giorgi, E.: Sulla proprietà isoperimetrica dell'ipersfera, nella classe degli insiemi aventi frontiera orientata di misura finita. Atti Accad. Naz. Lincei. Mem. Cl. Sci. Fis. Mat. Nat. Sez. I(8), 33-44 (1958)

8. Frank, R.L., Lieb, E.H.: A compactness lemma and its application to the existence of minimizers for the liquid drop model. SIAM J. Math. Anal. 47(6), 4436-4450 (2015)

9. Gamow, G.: Mass defect curve and nuclear constitution. Proc. R. Soc. Lond. Ser. A 126, 632-644 (1930)

10. Knüpfer, H., Muratov, C.: On an isoperimetric problem with a competing nonlocal term II: the general case. Commun. Pure Appl. Math. 67(12), 1974-1994 (2014)

11. Knüpfer, H., Muratov, C., Novaga, M.: Low density phases in a uniformly charged liquid (2015). arXiv:1504.05600 (preprint)

12. Lu, J., Otto, F.: Nonexistence of minimizers for Thomas-Fermi-Dirac-von Weizsäcker model. Commun. Pure Appl. Math. 67(10), 1605-1617 (2014)

13. Maggi, F.: Sets of finite perimeter and geometric variational problems. In: An Introduction to Geometric Measure Theory. Cambridge Studies in Advanced Mathematics, vol. 135. Cambridge University Press, Cambridge (2012)

14. Nam, P.T., Van Den Bosch, H.: Nonexistence in Thomas-Fermi-Dirac-von Weizsäcker theory with small nuclear charges (2016). arXiv:1603.07368 (preprint)

15. Riesz, F.: Sur une inégalité intégrale. J. Lond. Math. Soc. 5, 162-168 (1930)

16. Weizsäcker, C.F.von : Zur Theorie der Kernmassen. Zeitschrift für Physik A 96, 431458 (1935) 